\title{
Extrapulmonary Tuberculosis: Update on the Epidemiology, Risk Factors and Prevention Strategies
}

\author{
Houda Ben Ayed ${ }^{1,2^{*}}$, Makram Koubaa ${ }^{2,3^{*}}$, Chakib Marrakchi ${ }^{2,3}$, Khaoula Rekik ${ }^{2,3}$, Fatma \\ Hammami $^{2,3}$, Fatma Smaoui i,3, Mariem Ben Hmida ${ }^{1}$, Sourour Yaich ${ }^{1}$, Imed Maaloul2,3, \\ Jamel Damak ${ }^{1}$ and Mounir Ben Jemaa ${ }^{2,3}$
}

${ }^{1}$ Community Health and Epidemiology Department, Hedi Chaker University Hospital, University of Sfax, Tunisia

${ }^{2}$ Extrapulmonary Research Unit, Hedi Chaker University Hospital, Tunisia

${ }^{3}$ Infectious Diseases Department, Hedi Chaker University Hospital, University of Sfax, Tunisia

*Corresponding author: Houda Ben Ayed, MD, Community Health and Epidemiology Department, Hedi Chaker University Hospital, University of Sfax, Tunisia

Makram Koubaa, MD, Infectious Diseases Department, Hedi Chaker University Hospital, University of Sfax, Tunisia, Tel: $+216-21-880-402$

\begin{abstract}
Tuberculosis (TB) is one of the major public health threats, competing with the human immunodeficiency virus (HIV) as the cause of death due to infectious diseases worldwide. Extrapulmonary tuberculosis (EPTB) contributes to the burden of disease and does not receive specific attention in international control strategies. We aimed to give an update on the current epidemiological data of EPTB, to describe its clinical spectrum, to identify the main risk factors and to propose effective preventive strategies to reduce EPTB burden. Recent epidemiological data showed that the proportion of EPTB are increasing in different countries, accounting for $15 \%$ of the 6.3 million incident cases of TB that were notified in 2016 worldwide. The commonest EPTB reported sites were lymph node, pleura and urogenital tuberculosis. EPTB site has been associated with the immune status of TB patients. The main reported risk factors of EPTB were HIV infection and low CD4 count, female gender, extremes of age, poverty and several host genes. The main way to stop the transmission of this disease is implementation of effective preventive strategies. They consist of early diagnosis and treatment of active TB, the prevention of active disease in latently infected individual's, vaccination and ensure continued surveillance for infected patients as well as active controlling of zoonotic TB. The key to achieving the STOP TB target of global TB eradication by 2050 will be sustained commitment from donors, authorities, effective national TB programs as well as community engagement.
\end{abstract}

\section{Keywords}

Epidemiology, Extra-pulmonary tuberculosis, Prevention, Risk factor

\section{Introduction}

Tuberculosis (TB) is one of the major public health threats, competing with the human immunodeficiency virus (HIV) as the cause of death due to infectious diseases worldwide. TB is the ninth leading cause of death worldwide and the leading cause from a single infectious agent, ranking above HIV/AIDS [1]. In 2016, an estimated 10.4 million people fell ill with TB among whom $56 \%$ were living in five countries: India, Indonesia, China, the Philippines and Pakistan [1]. TB is a multisystem disease with myriad presentations and manifestations that typically affect the lung, but can also affect any organ or tissue, excluding only the hair and nails. Although pulmonary tuberculosis (PTB) accounts for the majority of cases and is the main transmissible form of the disease, extrapulmonary tuberculosis (EPTB) also contributes to the burden of disease and does not receive specific attention in international control strategies [2]. We aimed, through this review, to give an update on the epidemiology, the risk factors and the main prevention strategies of EPTB.

\section{Review of Current Epidemiological Data}

EPTB represented $15 \%$ of the 6.3 million incident cases that were notified in 2016, ranging from $8 \%$ in the WHO Western Pacific Region to $24 \%$ in the WHO East- 
ern Mediterranean Region [1]. In Sub-Saharan Africa, North America and in Australia EPTB had exceeded 30\% of all new and relapse tuberculosis cases in 2016 [1]. In Cambodia, a high burden-country of TB $(222 / 100,000$ populations), it has been estimated that EPTB accounted for $34 \%$ of all TB cases. Of these, $16 \%$ were children aged under 15 years [1]. EPTB rates varied widely according to the socio-economic level of the country and to the resources devoted to TB programs. The proportion of EPTB among all the reported tuberculosis cases has experienced an increase in different populations as well, with the rise of HIV epidemic in many regions of the world accounting for $19.3 \%$ (5.8 to $44.4 \%$ ) of all TB notified cases in the European Union, $18.7 \%$ in the USA and $12 \%$ in Brazil [3-5]. In contrast to PTB, EPTB rates are not decreasing: The proportion of EPTB increased from $16.4 \%$ in 2002 to $22.4 \%$ in 2011 in the European Union [3]. According to the Center for Disease Control and Prevention (CDC), the proportion of EPTB among all TB cases remained high, and the overall decline in TB cases since 1953 has been almost exclusively due to a reduction in pulmonary TB and not to EPTB [6]. PetoHM demonstrated that the proportion of EPTB cases in the USA has been rising from $15.7 \%$ in 1993 to $21 \%$ in 2006 due to a slower rate of decline in EPTB compared to PTB [4]. Likewise, in Australia, EPTB makes up over 50\% and this proportion has been reportedly increasing in many Western regions as well $[4,7]$. The National Surveillance System of TB in Germany reported that the mean annual incidence rate of notified TB was 6.3/100,000 population from 2002 to 2009 and that $30.8 \%$ patients had EPTB of patients with information on the anatomic site of the disease [8].

However, EPTB is largely undiagnosed in developing countries due to the difficulties in its diagnosis, especially when visceral sites are involved. Thus, EPTB deserves an increasing focus in these areas. Previous studies conducted in Oman, India and Saudi Arabia showed that EPTB patients accounted for $37 \%, 16 \%$ and $57.5 \%$ of all notified TB cases, respectively [9-11]. A recent study conducted in South Africa showed a significant decrease of $13 \%$ in overall number of TB, while TB meningitis and TB of bones increased significantly and TB pleural effusion and lymphadenopathy remained the same [12]. A study conducted in Cameroon including 984 patients in 2010 showed that about a quarter of patients with PTB have also EPTB (26.6\%) [13].

\section{Extrapulmonary Tuberculosis Tropism}

The commonest EPTB reported sites were lymph node, pleura and urogenital tuberculosis $[2,14,15]$. Lymphatic system accounted for $28 \%$ in USA [16], 39.4\% in Turkey [15] and 31\% in South Africa [14] of all EPTB cases. In some countries, TB of bone and joints had reached the leading position among EPTB cases, such as in Russia (34.5\%) [2] and in Taiwan (24.5\%) [17]. Among 141 EPTB patients in Istanbul over 7 years, TB of the central ner- vous system accounted for $23 \%$, and TB lymphadenitis for $21 \%$. Other types of EPTB were musculoskeletal system, miliary, peritoneal, abscess, urogenital, cutaneous and gastrointestinal involvement which ranged between $1 \%$ and $18 \%$ [18]. In two provinces in Turkey, pleural TB was the most involved EPTB site (41.4\%), followed by lymphatic (25.7\%) and genitourinary system (7.8\%) [19]. In countries with epidemic TB, urogenital tuberculosis is the most common form among EPTB, while in countries with low incidence rates, it is revealed to be rare [20]. It has been estimated that the proportion of patients with concomitant PTB ranged from 2 and 10\% of patients in developed countries and increased up to $15-20 \%$ in developing countries [21]. Another Omani study conducted between 2006 and 2015 showed that the most common sites of EPTB were the lymph nodes (42\%) and the abdomen (31\%), followed by brain (10\%) and musculoskeletal tissue (10\%) [9]. In fact, EPTB site has been associated with the immune status of TB patients, since there is increased susceptibility for reactivation and dissemination of tuberculosis in these patients. Among patients hospitalized with EPTB, those coinfected with HIV and low CD4 counts $\left(<100 / \mathrm{mm}^{3}\right)$ were more likely to have "severe" sites of disease such as meningeal and disseminated TB (Adjusted OR 1.6; 95\% Cl 1.0, 2.4), compared to lymphatic EPTB. Furthermore, patients with concomitant pulmonary tuberculosis were more likely to have disseminated EPTB (Adjusted OR 1.9, 95\% $\mathrm{Cl} 1.3,2.8)$ [16]. The spectrum of EPTB is large and severe forms are a grave threat to patients' health. Therefore, investigating EPTB determinants and identifying patients at higher risk of EPTB involvement is urgently needed in order to improve TB management and to ameliorate the diseases prognosis.

\section{Extrapulmonary Tuberculosis Risk Factors}

Understanding the risk factors of EPTB is a crucial step to target the preventive strategies and to address authorities to implement effective interventions accordingly. These factors are myriad and need more attention to pay due to variation in the pathogenesis of each EPTB site.

\section{Immunological risk factors}

HIV infection is the greatest risk factor for the development of EPTB disease in persons with latent TB, due to a weakened immune system. The risk of TB progression is much higher at about $10 \%$ per year in HIV-positive and other immune-compromised individuals and who are not receiving highly active treatment for HIV [22]. A systematic review of 16 studies from 1984 to 2016 studying the association of HIV infection and EPTB showed increased odds of EPTB compared with PTB among HIV-infected individuals [23]. The close association between HIV infection and EPTB is very likely due to deficiency of CD4+ T cells among HIV infected patients. It is well known that HIV targets on CD4+ T cells and causes reduced CD4+ T cells and less cytokine pro- 
duction. CD4+ T-helper cells are major players for controlling M. tuberculosis infection [24]. Similarly, Naing $C$ reported that EPTB in HIV patients was significantly associated with CD4 counts less than 100 , with an OR of 1.3; (95\% Cl 1.05-1.6) [25]. Close to $14 \%$ to $56 \%$ of HIV patients had extrapulmonary, miliary or disseminated tuberculosis [26].

\section{Socio-demographic risk factors}

It has been reported that host risk factors for EPTB include younger age, female sex and non-white race $[27,28]$. Another study conducted in Brazil including 606 patients showed that illiteracy, ethnicity and HIV were associated with EPTB [5]. A large-scale study conducted in Spain from 2007 to 2012 showed that he main EPTB risk factor was presence of HIV infection (OR 1.39) and that persons aged over 65 years as well as foreign-born patients had a higher risk of EPTB [9]. A population-based case-control study in the United States showed that $16.9 \%$ of female patients had extrapulmonary disease compared to $9.3 \%$ of male patients [27]. Hormonal factors, smoking and TB exposure might be the causes of this inequality [29]. Older women are less able to contain bacilli in the lungs due to reduced levels of sex hormones after menopause [30]. On the other hand, it has been reported that patients with EPTB diagnosis were more likely to be 65 years or older, to be retired, and to live in urban areas [31]. Previous studies showed that female gender (OR 1.6-2.04) advanced age (OR 1.02-2.1), having end-stage renal disease and semi-skilled occupation were independently associated with EPTB patients $[17,27,32,33]$. A population-based case-control study, showed that the risk of EPTB among younger than 25-years-old is 2-fold of that among older patients [27]. Similar results were found in other studies from Europe and USA, where younger age was independently associated with EPTB $[34,35]$.

\section{Comorbidities and lifestyle behaviors}

Previous studies illustrated that the reactivation of tuberculosis seems to occur at least four years after the initial diagnosis of diabetes [26] and that the risk of death was high in diabetic patients with EPTB [36]. It has been reported that previous liver disease (OR = 22.30; 95\% IC: 1.89 - 263.57) was an independent risk factor for peritoneal tuberculosis and that HIV co-infection (OR $=12.97 ; 95 \%$ IC: $1.71-48.42$ ) and the presence of previous TB treatment (OR $=7.62 ; 95 \%$ IC: $1.00-57.9$ ) increased the risk of disseminated disease [37]. When compared to PTB patients, patients with EPTB were less likely to smoke [9].

\section{Genetic factors}

Additionally, it was well known that several host genes were involved in tuberculosis dissemination: TLR2 genotype T597C was found to be associated with TB meningitis in a case-control study and increased the severity of neurologic symptoms [38]. In this same point of view, genetic polymorphisms in interleukin were associated with pleural TB [39].

\section{Lymph node pathogenesis}

Concerning lymph node TB, the pathogenesis is quite different, since it is most frequently transmitted from infected animals. In consumed raw milk, the identification of $M$. bovis and atypical mycobacteria has been described in Tanzania, M. bovis and $M$. africanum in $\mathrm{Ni}$ geria, M. bovis in Tunisia, and atypical Mycobacterium species in Turkey and Brazil [40]. After invasion by oral or respiratory routes, the pathogen is actively phagocytosed by macrophages. Then, it is captured in regional lymph nodes and causes clinical manifestations of lymph node TB.

\section{Extrapulmonary Tuberculosis Prevention Strate- gies}

\section{The WHO framework}

With a vision to progress towards finally eliminating tuberculosis, the World Health Organization (WHO) has developed a global TB strategy with a perspective beyond 2015. It includes, a goal to reduce global TB incidence from $>1000$ cases per million population today to $<100$ cases per million by 2035 [41]. In low-incidence countries, a framework has been implemented to prioritize interventions required for these countries to progress first towards "pre-elimination" $1<10$ cases per million) and eventually the elimination of TB as a public health problem ( $<1$ case per million). This framework presented a tailored response to these challenges, grouped into eight priority action areas: 1) Ensure political commitment, funding and stewardship for planning and essential services; 2) Address the most vulnerable and hard-to-reach groups; 3 ) Address special needs of migrants and cross-border issues; 4) Undertake screening for active TB and latent tuberculosis infection in TB contacts and selected high-risk groups, and provide appropriate treatment; 5) Optimize the prevention and care of drug-resistant TB; 6) Ensure continued surveillance, program monitoring and evaluation and casebased data management; 7) Invest in research and new tools and 8) Support global TB prevention, care and control. The overall approach needs to be multisectoral, focusing on equitable access to high-quality diagnosis and care, and on addressing the social determinants of TB. Because of increasing globalization and population mobility, the response needs to have both national and global dimensions [42].

EPTB is generally not associated with person-to-person spread. Prevention is the key to stop its transmission. It consists of early diagnosis and treatment of active TB to stop infectiousness, the prevention of active disease in latently infected individuals and vaccination.

\section{Treatment of latent tuberculosis infection}

Treatment of individuals with active tuberculosis 
(TB) is the first priority for TB control; but an important second priority is identification and treatment of individuals with latent tuberculosis in order to avoid the disease dissemination. The WHO recommends that chemoprophylaxis of these patients must be included as part of the TB control program for high income or upper middle-income countries with an estimated TB incidence rate of less than 100 per 100,000 population per year [43]. For treatment of latent tuberculosis infection in HIV-uninfected adults, the WHO suggested either nine months of self-administered isoniazid (INH) or three months of weekly INH and rifapentine (RPT), preferably administered via directly observed therapy. Alternative regimens include daily INH for six months, daily rifampin (RIF) for four months, or daily INH and RIF for three months [44]. For HIV-infected patients with latent $\mathrm{TBC}$, they are significantly more likely to reactivate with TB disease than HIV-uninfected individuals. Treatment of latent TB is associated with two important benefits: Firstly, reduction in the likelihood of progression to active TB disease, and secondly reduction in TB transmission. Chemoprophylaxis is warranted for HIV-infected patients in the following circumstances: Individuals with recent contact with a person with active TB disease, individuals with a history of inadequately treated healed TB (fibrotic disease on chest radiograph), regardless of test results for latent $T B$, individuals with evidence of latent TB by tuberculin skin test or interferon-gamma release assay and individuals living in resource-limited areas of high TB incidence where testing for latent TB is not available [44].

\section{BCG vaccination}

Currently, the only means of immunizing against TB is with the live attenuated vaccine BCG, although other vaccines are under development. Immunology studies supported the rationale for targeting prevention of infection, with evidence that host responses may be more effective during acute infection than during chronic infection [45]. Therefore, development of an effective TB vaccine is a primary global to reduce the rate of reactivation. A systematic review and a metanalysis studying the current evidence of BCG against tuberculosis showed that BCG vaccination efficacy was usually high, notably against serious forms of EPTB such as meningeal and miliary tuberculosis [46]. Reviewed studies have also shown high levels of protection against death in EPTB patients [46]. When BCG vaccination was given only to infants or to children after strict screening for tuberculin sensitivity, efficacy against EPTB was high compared to adults. BCG vaccination protects against EPTB for up to 10 years [46], but highly variable and often very low efficacy against PTB in adults was reported [47]. Thus, even with the high coverage now achieved, BCG is unlikely to have any substantial effect on transmission. In tuberculosis high-incidence countries, BCG vaccination is recommended for children at birth or at first contact with health services, whereas in low-incidence coun- tries, vaccination is being discontinued because of the low risk of infection and the confounding interpretation of tuberculin skin tests used to track persons infected during occasional outbreaks [47].

Another essential point must be surmised in EPTB control: Prevention strategies must also target on bovine tuberculosis, which is caused by Mycobacterium bovis that spreads from animal to human and resulted in lymph node TB [48]. This occurs mainly through the consumption of unpasteurized dairy products, but also less commonly through the consumption of raw or uncooked meat or direct physical contact with infected animals [48]. Thus, heat-treatment of milk and basic surveillance of zoonotic TB are the key to reducing the risk to people infection.

\section{Conclusion}

Despite the availability of a cure and knowledge on prevention of transmission, EPTB remains a pressing public health problem for a significant proportion of the world. In developing countries, EPTB is less well addressed by programs than PTB, while its identification is important for optimizing care. Therefore, investigating EPTB determinants and identifying patients at higher risk of EPTB involvement is urgently needed in order to improve TB management and to ameliorate the diseases prognosis. TB dissemination and acquiring EPTB form depend on a multitude of factors: Co-morbidities, HIV-coinfection, host factors, genetic variance and the site of the infection. Given the current alarming rates of EPTB burden, elimination of the disease at global level is still out of reach. Therefore, prevention of TB spreading must be a public health priority worldwide and massive resource investment is required. Poverty reduction has been emphasized by the United Nations as a tool to reduce TB burden. The key towards achieving the STOP TB target of global TB eradication by 2050 will be sustained commitment from donors, authorities, effective national TB programs as well as community engagement, which played a pivotal role in identifying the most vulnerable groups, assessing their specific needs and promoting good quality of life for TB patients.

\section{Conflict of Interest}

The authors declared that there is no conflict of interests.

\section{References}

1. WHO (2017) Global Tuberculosis Report 2017.

2. Kulchavenya E (2014) Extrapulmonary tuberculosis: Are statistical reports accurate? Therapeutic Advances in Infectious Disease 2: 61-70.

3. Sandgren A, Hollo V, Van der Werf MJ (2013) Extrapulmonary tuberculosis in the European Union and European Economic Area, 2002 to 2011. Euro Surveill 18: 20431.

4. Peto HM, Pratt RH, Harrington TA, LoBue PA, Armstrong LR (2009) Epidemiology of extrapulmonary tuberculosis in the United States, 1993-2006. Clin Infect Dis 49: 1350-1357. 
5. Gomes T, Vinhas SA, Reis-Santos B, Palaci M, Peres RL, et al. (2013) Extrapulmonary tuberculosis: Mycobacterium tuberculosis strains and host risk factors in a large urban setting in Brazil. PLoS One 8: e74517.

6. Adada H, Valley M, Nour S, Mehta J, Byrd R, et al. (2014) Epidemiology of extra-pulmonary tuberculosis in the United States: High rates persist in the post-HIV era. Int $\mathrm{J}$ Tuberc Lung Dis 18: 1516-1521.

7. Barry C, Waring J, Stapledon R, Konstantinos A (2012) Tuberculosis notifications in Australia, 2008 and 2009. Commun Dis Intell Q Rep 36: 82-94.

8. Ducomble T, Tolksdorf K, Karagiannis I, Hauer B, Brodhun B, et al. (2013) The burden of extrapulmonary and meningitis tuberculosis: An investigation of national surveillance data, Germany, 2002 to 2009. Euro Surveill 18: 20436.

9. Gaifer Z (2017) Epidemiology of extrapulmonary and disseminated tuberculosis in a tertiary care center in Oman. Int J Mycobacteriol 6: 162-166.

10. (2014) Tuberculosis control in the South-East Asia region: Annual TB report 2014. WHO Regional Office for SouthEast Asia.

11. Al-Otaibi F, El Hazmi MM (2010) Extra-pulmonary tuberculosis in Saudi Arabia. Indian J Pathol Microbiol 53: 227-231.

12. Hoogendoorn J, Ranoto L, Muditambi N, Railton J, Maswanganyi M, et al. (2017) Reduction in extrapulmonary tuberculosis in context of antiretroviral therapy scale-up in rural South Africa. Epidemiol Infect 145: 2500-2509.

13. Pefura Yone EW, Kengne AP, Moifo B, Kuaban C (2013) Prevalence and determinants of extrapulmonary involvement in patients with pulmonary tuberculosis in a Sub-Saharan African country: A cross-sectional study. Scand J Infect Dis 45: 104-111.

14. Karstaedt A (2014) Extrapulmonary tuberculosis among adults: Experience at Chris Hani Baragwanath Academic Hospital, Johannesburg, South Africa. South African Medical Journal 104: 22-24.

15. Sunnetcioglu A, Sunnetcioglu M, Binici I, Baran AI, Karahocagil MK, et al. (2015) Comparative analysis of pulmonary and extrapulmonary tuberculosis of 411 cases. Ann Clin Microbiol Antimicrob 14: 34.

16. Leeds IL, Magee MJ, Kurbatova EV, del Rio C, Blumberg $\mathrm{HM}$, et al. (2012) Site of extrapulmonary tuberculosis is associated with HIV infection. Clin Infect Dis 55: 75-81.

17. Lin J, Lai C, Chen Y, Lee S, Tsai S, et al. (2009) Risk factors for extra-pulmonary tuberculosis compared to pulmonary tuberculosis. Int J Tuberc Lung Dis 13: 620-625.

18. Sevgi DY, Derin O, Alpay AS, Gündüz A, Konuklar AS, et al. (2013) Extrapulmonary tuberculosis: 7 year-experience of a tertiary center in Istanbul. Eur J Intern Med 24: 864-867.

19. Tatar D, Senol G, Alptekin S, Gunes E, Aydin M, et al. (2016) Assessment of extrapulmonary tuberculosis in two provinces of Turkey. Iran J Public Health 45: 305-313.

20. Kulchavenya E, Krasnov V (2012) Diseases of urinary bladder. Nauka (Science), Novosibirsk, 187.

21. Figueiredo AA, Lucon AM (2008) Urogenital tuberculosis: Update and review of 8961 cases from the world literature. Rev Urol 10: 207-217.

22. Narasimhan P, Wood J, Maclntyre CR, Mathai D (2013) Risk factors for tuberculosis. Pulm Med 2013: 828939.

23. Shivakoti R, Sharma D, Mamoon G, Pham K (2017) Association of HIV infection with extrapulmonary tuberculosis: A systematic review. Infection 45: 11-21.
24. Yang D, Kong Y (2015) The bacterial and host factors associated with extrapulmonary dissemination of Mycobacterium tuberculosis. Front Biol 10: 252-261.

25. Naing C, Mak JW, Maung M, Wong SF, Kassim AI (2013) Meta-analysis: The association between HIV infection and extrapulmonary tuberculosis. Lung 191: 27-34.

26. Nantha S (2014) A review of tuberculosis research in Malaysia. Med J Malaysia 69: 88-102.

27. Yang Z, Kong $Y$, Wilson F, Foxman B, Fowler AH, et al. (2004) Identification of risk factors for extrapulmonary tuberculosis. Clin Infect Dis 38: 199-205.

28. Fiske CT, Griffin MR, Erin H, Warkentin J, Lisa K, et al. (2010) Black race, sex, and extrapulmonary tuberculosis risk: An observational study. BMC Infectious Diseases 10: 16.

29. Chiang C, Slama K, Enarson D (2007) Associations between tobacco and tuberculosis [Educational Series: Tobacco and tuberculosis. Serialised guide. Tobacco cessation interventions for tuberculosis patients. Number 1 in the series]. Int J Tuberc Lung Dis 11: 258-262.

30. Holmes C, Hausler H, Nunn P (1998) A review of sex differences in the epidemiology of tuberculosis. Int $\mathrm{J}$ Tuberc Lung Dis 2: 96-104.

31. Wang X, Yang Z, Fu Y, Zhang G, Wang X, et al. (2014) Insight to the epidemiology and risk factors of extrapulmonary tuberculosis in Tianjin, China during 2006-2011. PLoS One 9: e112213.

32. García-Rodríguez JF, Álvarez-Díaz H, Lorenzo-García MV, Mariño-Callejo A, Fernández-Rial Á, et al. (2011) Extrapulmonary tuberculosis: Epidemiology and risk factors. Enferm Infecc Microbiol Clin 29: 502-509.

33. Memish ZA, Bamgboye EA, Abuljadayel N, Smadi H, Abouzeid MS, et al. (2014) Incidence of and risk factors associated with pulmonary and extra-pulmonary tuberculosis in Saudi Arabia (2010-2011). PLoS One 9: e95654.

34. Gonzalez O, Adams G, Teeter L, Bui T, Musser JM, et al. (2003) Extra-pulmonary manifestations in a large metropolitan area with a low incidence of tuberculosis. Int $\mathrm{J}$ Tuberc Lung Dis 7: 1178-1185.

35. Cailhol J, Decludt B, Che D (2005) Sociodemographic factors that contribute to the development of extrapulmonary tuberculosis were identified. J Clin Epidemiol 58: 10661071.

36. Magee M, Foote M, Ray S, Gandhi N, Kempker R (2016) Diabetes mellitus and extrapulmonary tuberculosis: Site distribution and risk of mortality. Epidemiol Infect 144: 2209-2216.

37. Sanches I, Carvalho A, Duarte R (2015) Who are the patients with extrapulmonary tuberculosis? Revista Portuguesa de Pneumologia 21: 90-93.

38. Thuong N, Hawn T, Thwaites G, Chau T, Lan N, et al. (2007) A polymorphism in human TLR2 is associated with increased susceptibility to tuberculous meningitis. Genes Immun 8: 422-428.

39. Wilkinson RJ, Patel P, Llewelyn M, Hirsch CS, Pasvol G, et al. (1999) Influence of polymorphism in the genes for the interleukin (IL)-1 receptor antagonist and IL-1 $\beta$ on tuberculosis. J Exp Med 189: 1863-1874.

40. Bolaños CAD, Paula CLd, Guerra ST, Franco MMJ, Ribeiro MG (2017) Diagnosis of mycobacteria in bovine milk: An overview. Rev Inst Med Trop São Paulo 59: e40. 
41. Raviglione MC, Ditiu $L$ (2013) Setting new targets in the fight against tuberculosis. Nat Med 19: 263.

42. Lönnroth K, Migliori GB, Abubakar I, D'Ambrosio L, De Vries G, et al. (2015) Towards tuberculosis elimination: An action framework for low-incidence countries. Eur Respir J 45: 928-952.

43. Getahun H, Matteelli A, Abubakar I, Aziz MA, Baddeley A, et al. (2015) Management of latent Mycobacterium tuberculosis infection: WHO guidelines for low tuberculosis burden countries. Eur Respir J 46: 1563-1576.

44. WHO (2018) Latent TB Infection: Updated and consolidated guidelines for programmatic management.

45. Hawn TR, Day TA, Scriba TJ, Hatherill M, Hanekom WA, et al. (2014) Tuberculosis vaccines and prevention of infection. Microbiol Mol Biol Rev 78: 650-671.

46. Abubakar I, Pimpin L, Ariti C, Beynon R, Mangtani P, et al. (2013) Systematic review and meta-analysis of the current evidence on the duration of protection by bacillus CalmetteGuérin vaccination against tuberculosis. Health Technol Assess 17: 1-372.

47. Jamison DT, Breman JG, Measham AR, Alleyne G, Claeson $\mathrm{M}$, et al. (2006) Disease control priorities in developing countries. $\left(2^{\text {nd }}\right.$ edn $)$,World Bank Publications, ISBN-10: 0-8213-6179-1.

48. WHO (2018) The challenges of preventing bovine tuberculosis. Bull World Health Organ 96: 82-83. 